\title{
Screening for COVID-19 in Children Undergoing Elective Invasive Procedures
}

\author{
Briseida López-Martinez ${ }^{1}$. Gerardo Blanco-Rodríguez ${ }^{2}$ Israel Parra-Ortega ${ }^{1}$ - Cesar Emmanuel Ramirez-Martinez ${ }^{2}$. \\ Miguel Angel Villasis-Keever ${ }^{3} \cdot$ Maria Guadalupe Miranda-Novales ${ }^{3} \cdot$ Miguel Klünder-Klünder $^{4}$. \\ Daniela De la Rosa Zamboni ${ }^{5}$. Monica Villa Guillen ${ }^{6}$. Jaime Nieto-Zermeño ${ }^{6}$ Juan Garduño-Espinosa ${ }^{7}$. \\ Jessie Nallely Zurita-Cruz ${ }^{8}$
}

Received: 28 May 2021 / Accepted: 5 August 2021/Published online: 22 October 2021

(c) Dr. K C Chaudhuri Foundation 2021

\begin{abstract}
Objective To report the frequency of asymptomatic infection with SARS-CoV-2 in pediatric patients undergoing invasive medical procedures in a tertiary pediatric hospital.

Methods From June to October 2020, a SARS-CoV-2 real-time reverse-transcription polymerase chain reaction (rRT-PCR) test was performed for all pediatric patients scheduled to undergo an elective invasive procedure. None of the patients was symptomatic. The cycle threshold $(\mathrm{Ct})$ values of the ORFlab gene were recorded for all patients.

Results A total of 700 patients were screened for SARS-CoV-2 infection. The median age was $5.7 \mathrm{y}$ old. In total, $46.6 \%$ $(n=326)$ of the patients were male, and $53.4 \%(n=374)$ were female. The most common underlying diseases were hematooncological (25.3\%), gastrointestinal (24.9\%), and genitourinary (10.3\%). The main scheduled surgical-medical procedures were surgical treatment for acquired congenital diseases, biopsy sampling, local therapy administration, organ transplantation, and the placement of central venous catheters, among others. The SARS-CoV-2 rRT-PCR test was positive in 9.4\% (66), and the median $\mathrm{Ct}$ value was 35.8. None of the patients developed COVID-19.

Conclusions The frequency of asymptomatic SARS-CoV-2 infection was detected in less than $10 \%$ of pediatric patients scheduled to undergo an elective invasive procedure in a tertiary hospital. This frequency is higher than those in reports from different countries.
\end{abstract}

Keywords SARS-CoV-2 $\cdot$ COVID-19 $\cdot$ Asymptomatic $\cdot$ Children

Jessie Nallely Zurita-Cruz

zuritajn@hotmail.com

1 Auxiliary Diagnostic Services, Hospital Infantil de México Federico Gómez, Ministry of Health (SSA), Mexico City, Mexico

2 Thoracic Surgery and Endoscopy Service, Hospital Infantil de México Federico Gómez, Ministry of Health (SSA), Mexico City, Mexico

3 Analysis and Synthesis of the Evidence Research Unit, National Medical Center XXI Century, Instituto Mexicano del Seguro Social, Mexico City, México

4 Medical Research Direction, Hospital Infantil de México Federico Gómez, Ministry of Health (SSA), Mexico City, Mexico
5 Epidemiology Services, Hospital Infantil de México Federico Gómez, Ministry of Health (SSA), Mexico City, Mexico

6 Medical Director, Hospital Infantil de México Federico Gómez, Ministry of Health (SSA), Mexico City, Mexico

7 Hospital Director, Hospital Infantil de México Federico Gómez, Ministry of Health (SSA), Mexico City, Mexico

8 Facultad de Medicina, Universidad Nacional Autónoma de México, Hospital Infantil de México Federico Gómez, Mexico City 06720, Mexico 


\section{Introduction}

In the last months of 2019, a new coronavirus was identified as the cause of a severe form of pneumonia in China. Coronavirus disease 2019 (COVID-19) is caused by severe acute respiratory syndrome coronavirus 2 (SARS-CoV-2) [1-4].

There is controversy about the role of children and young adults in the spread of SARS-CoV-2. It seems that children could develop a mild upper respiratory infection after infection with SARS-CoV-2 with low viral shedding, indicating that they are seldom responsible for secondary cases, as reported by Zhu et al. [2, 5].

A systematic review showed that children accounted for $1 \%-5 \%$ of all confirmed cases of COVID-19, and most of them presented with mild disease, in contrast with the adult population [6]. Even when cases of the disease are mild, transmission of the virus is possible [7]. According to the data from a study in Korea that included 91 children younger than $19 \mathrm{y}, 22 \%$ of the children with a positive test did not develop symptoms of disease, $20 \%$ were in the presymptomatic period, and 58\% were symptomatic. Tests to detect SARS-CoV-2 were repeatedly performed in children to determine the duration of positivity for virus RNA. Overall, SARS-CoV-2 RNA was detected for a mean (SD) of 17.6 (6.7) d. In patients with asymptomatic cases, viral RNA was detected for a mean of 14.1 (7.7) d, while symptomatic children with upper and lower respiratory tract infections were positive for a mean of 18.7 (5.8) d and 19.9 (5.6) d, respectively [8].

Since the beginning of the pandemic, surgical societies and the College of Surgeons have recommended screening for SARS-CoV-2 infection in all patients scheduled to undergo surgery. The incubation period has been estimated to be 5.5 $\mathrm{d}$, and more than $90 \%$ of subjects who test positive for viral RNA are expected to develop symptoms 11 to $12 \mathrm{~d}$ after exposure [9]. In the context of community transmission, it must be assumed that every patient could be infected with SARS$\mathrm{CoV}-2$, even if the answers to the screening questions are negative, and the patient's body temperature is normal [10].

Children with chronic diseases require ongoing medical care, and disruption in their planned treatment could have serious consequences. Therapeutic and diagnostic procedures need to be performed to ensure the safety of the patients and medical staff [11].

The objective of this study was to report the frequency of asymptomatic infection with SARS-CoV-2 in pediatric patients undergoing elective invasive procedures in a tertiary pediatric hospital.

\section{Material and Methods}

This cross-sectional study was performed from June 2020 to October 2020 at a national tertiary pediatric referral center in Mexico City. For the writing of the study, the
STROBE criteria were followed. Patients from 1 mo to $18 \mathrm{y}$ of age whose parents provided informed consent and who visited the outpatient clinic and underwent an elective invasive procedure were included. The elective procedures were surgical treatments for acquired congenital diseases, biopsy sampling, local therapy administration (such as intrathecal therapy), solid organ transplantation, and the placement of central venous catheters, among others.

In March 2020, the Mexican government implemented preventive measures to contain the pandemic; thus, in this tertiary pediatric hospital, only patients with suspected cases of COVID-19 were treated, and the rest of the patients were referred to other hospitals. In June 2020 , routine activities were gradually resumed. Each week, more patients who had been previously treated in the hospital returned, and in August, new patients were also accepted. As part of the protocol implemented in the hospital, all patients undergoing elective invasive procedures were screened at the first medical visit with questions about COVID-19 symptoms, close contact with confirmed cases in the previous $14 \mathrm{~d}$, and exposure locations (if any). If the patient had no identifiable risk factors for SARS-CoV-2 infection, real-time reverse-transcription polymerase chain reaction (rRT-PCR) was used to detect the presence of SARS-CoV-2. Patients with clinical symptoms were referred to the respiratory triage area for further evaluation. Patients with an unclear exposure history were rescheduled for re-evaluation in two weeks.

The results of the rRT-PCR tests for SARS-CoV-2 were provided to the patients over the phone during the first $24 \mathrm{~h}$ after the samples were taken. Patients with a negative result continued with the procedures as scheduled. For those with a positive result, home isolation was mandated; in the case of mild symptoms (fever, tiredness, sore throat, coughing, nasal congestion, muscle or body aches, headache, or diarrhea), they were instructed to communicate with the hospital's call center; in the case of severe symptoms (trouble breathing, rapid respiration rate, flushed lips or face, oral intolerance, signs of dehydration, or new confusion), they were instructed to go immediately to the hospital's emergency room. Fifteen days after the positive SARS-CoV-2 result, they were asked to return to the hospital for a full examination by the medical staff to determine whether they could continue with the elective procedures.

The protocol was evaluated and approved by the ethics and research committee of the hospital with registry number HIM-2020-023. The parent or legal guardian signed a written informed consent form, and each child provided written assent.

Infection with SARS-CoV-2 was detected by rRT-PCR with a combined nasopharyngeal and oropharyngeal swab sample. Total RNA was extracted and purified with the 
robot QIAcube (QIAGEN) and the QIAamp® Viral RNA mini kit (QIAGEN) according to the protocol of the manufacturer and the current operational guidelines established by the Mexican Institute of Epidemiological Diagnosis and Reference [12] (Supplementary material). The cycle threshold $(\mathrm{Ct})$ values of the ORFlab gene were recorded for all cases, with lower values indicating higher amounts of viral RNA.

For quantitative variables, Kolmogorov-Smirnov tests were performed to evaluate the normality of the distribution of the data; those with non-normal distributions are presented as medians and $95 \%$ confidence intervals. The patients were divided into two groups: those with COVID19 and those without COVID-19. Qualitative variables are presented as proportions and frequencies. STATA v.12.0 was used for the statistical analyses.

\section{Results}

Of the 777 potential candidates for inclusion, 77 were excluded because their parents refused to allow them to be tested for SARS-CoV-2. Of the 700 patients included in the study, the median age was $7.5 \mathrm{y}$, with a slight predominance of males (53.4\%). The most frequent underlying condition was hemato-oncological, followed by gastrointestinal and genitourinary (Table 1). The main type of surgical procedure or invasive medical procedure was the surgical correction of congenital deformities $(42.9 \%$, $n=300)$, followed by tumor resection surgery $(16.6 \%$, $n=116)$ and biopsy sampling $(11.7 \%, n=82)$ (Table 2$)$.
Of the 700 patients analyzed, $9.4 \%(n=66)$ had a positive test for SARS-CoV-2. In patients with a positive test for SARS-CoV-2, the main type of surgical procedure or invasive medical procedure was the surgical correction of congenital deformities $(45.5 \%, n=30)$, followed by tumor resection surgery $(28.8 \%, n=19)$ (Table 2$)$. All corresponded to elective procedures. In these patients, the surgery was deferred due to the positive report, but all the patients were medically stable, and procedures were performed as soon as possible after the follow-up evaluation. As shown in Fig. 1, the percentage of patients positive for SARS-CoV-2 varied over the $4 \mathrm{mo}$; the highest was in June (24.2\%), and the lowest was in October (4.6\%). The 66 positive patients were re-evaluated at a second visit to identify the possible source of infection, and $66.6 \%$ $(n=44)$ reported contact with a household member with a probable or confirmed case.None of the patients presented symptoms in the days after the positive test result.

In the 66 asymptomatic COVID-19 patients, the Ct values were high, with values ranging from 31.98 to 37.81 , indirectly indicating a low viral load. Across the months, the $\mathrm{Ct}$ values were similar (Fig. 1).

\section{Discussion}

Different strategies have been established in pediatric hospitals to ensure the safety of patients and hospital staff. In one hospital in England, children undergoing elective surgery were asked to isolate within their home for $14 \mathrm{~d}$ and then underwent rRT-PCR testing before surgery; of
Table 1 General characteristics of the included patients

\begin{tabular}{llll}
\hline & $\begin{array}{l}\text { Total } \\
n=700\end{array}$ & $\begin{array}{l}\text { Negative } \\
n=634\end{array}$ & $\begin{array}{l}\text { Positive } \\
n=66\end{array}$ \\
\cline { 2 - 4 } & Frequency $(\%)$ & & $5(3.5-8)$ \\
\hline Age (years)* & $7.5(7-8)$ & $8(7-8)$ & $30(45.4)$ \\
Female sex & $326(46.6)$ & $296(46.7)$ & $24(12-36)$ \\
Disease evolution time (months)* & $24(24-24)$ & $24(24-24)$ & \\
Type of pathology & & & $23(34.9)$ \\
Hemato-oncological & $177(25.3)$ & $154(24.3)$ & $17(25.8)$ \\
Gastrointestinal & $174(24.9)$ & $157(24.8)$ & $6(9.1)$ \\
Genitourinary & $72(10.3)$ & $66(10.4)$ & $2(3.0)$ \\
Cardiopulmonary & $64(9.1)$ & $62(9.8)$ & $1(1.5)$ \\
Otorhinolaryngeal & $53(7.6)$ & $52(8.2)$ & $8(12.1)$ \\
Neurological & $45(6.4)$ & $37(5.8)$ & $2(3.0)$ \\
Renal & $35(5.0)$ & $33(5.2)$ & $3(4.6)$ \\
Ophthalmic & $31(4.4)$ & $28(4.4)$ & $4(6.1)$ \\
Immunological & $25(3.6)$ & $21(3.3)$ & $0(0)$ \\
Musculoskeletal & $24(3.4)$ & $24(3.8)$ & $35.8(34.4-36.5)$ \\
Cycle threshold $(\mathrm{Ct})^{*}$ & & & \\
\hline
\end{tabular}

*Median (95\% confidence interval) 
Table 2 Type of surgical or invasive medical procedures according to SARS-CoV-2 rRTPCR test

\begin{tabular}{|c|c|c|c|}
\hline & $\begin{array}{l}\text { Total } \\
n=700\end{array}$ & $\begin{array}{l}\text { Negative } \\
n=634\end{array}$ & $\begin{array}{l}\text { Positive } \\
n=66\end{array}$ \\
\hline & \multicolumn{3}{|c|}{ Frequency $(\%)$} \\
\hline Surgical correction of congenital deformities & $300(42.9)$ & $270(42.6)$ & $30(45.5)$ \\
\hline Gastrointestinal & $91(30.3)$ & $77(12.1)$ & $14(21.2)$ \\
\hline Cardiopulmonary & $64(21.4)$ & $62(9.8)$ & $2(3.0)$ \\
\hline Genitourinary & $61(20.3)$ & $56(8.8)$ & $5(7.6)$ \\
\hline Otorhinolaryngeal & $36(12.0)$ & $35(5.5)$ & $1(1.5)$ \\
\hline Neurological & $34(11.3)$ & $26(4.1)$ & $8(12.1)$ \\
\hline Musculoskeletal & $14(4.7)$ & $14(2.2)$ & $0(0.0)$ \\
\hline Tumor resection surgery & $116(16.6)$ & $97(15.3)$ & $19(28.8)$ \\
\hline Central nervous system & $28(24.1)$ & $24(3.8)$ & $4(6.1)$ \\
\hline Osseous & $27(23.3)$ & $26(4.1)$ & $1(1.5)$ \\
\hline Abdominal & $24(20.7)$ & $21(3.3)$ & $3(4.5)$ \\
\hline Thyroid & $16(13.8)$ & $11(1.7)$ & $5(7.6)$ \\
\hline Others & $21(18.1)$ & $15(2.4)$ & $6(9.1)$ \\
\hline Biopsy sampling & $82(11.7)$ & $78(12.3)$ & $4(6.1)$ \\
\hline Oncological & $42(51.2)$ & $42(6.6)$ & $0(0.0)$ \\
\hline Immunological & $25(30.5)$ & $21(3.3)$ & $4(6.1)$ \\
\hline Neurological & $11(13.4)$ & $11(1.7)$ & $0(0.0)$ \\
\hline Others & $4(4.9)$ & $4(0.6)$ & $0(0.0$ \\
\hline Local therapy applications & $59(8.4)$ & $53(8.4)$ & $6(9.1)$ \\
\hline Laser application & $39(66.1)$ & $36(5.7)$ & $3(4.5)$ \\
\hline Sclerotherapy of esophageal varices & $20(33.9)$ & $17(2.7)$ & $3(4.5)$ \\
\hline Surgical treatment of acquired disease & $53(7.6)$ & $52(8.2)$ & $1(1.5)$ \\
\hline Otorhinolaryngeal & $41(77.4)$ & $41(6.5)$ & $0(0.0)$ \\
\hline Genitourinary & $11(20.7)$ & $10(1.6)$ & $1(1.5)$ \\
\hline Musculoeskeletal & $1(1.9)$ & $1(0.2)$ & $0(0.0)$ \\
\hline Kidney and liver transplantation & $29(4.1)$ & $28(4.4)$ & $1(1.5)$ \\
\hline Placement of central venous catheter & $22(3.1)$ & $21(3.3)$ & $1(1.5)$ \\
\hline Application of intrathecal therapy and biopsy & $16(2.3)$ & $12(1.9)$ & $4(6.5)$ \\
\hline Others & $23(3.3)$ & $23(3.6)$ & $0(0.0)$ \\
\hline
\end{tabular}

a total of 501 elective procedures, only $2.6 \%$ had to be cancelled due to SARS-CoV-2 infection [13]. In contrast, this study detected asymptomatic SARS-CoV-2 infections in $9.4 \%$ of the pediatric patients scheduled for an invasive procedure. In the present case, no recommendation to self-isolate was made, and Mexico has been experiencing sustained community transmission since April 21, 2020. Specific pediatric populations that need ongoing medical care have been screened for SARS-CoV-2 infection, as they cannot maintain a complete quarantine and need to
Fig. 1 Cycle threshold values (a) and proportion of patients positive for infection with SARS-CoV-2 (b) stratified by month and the percentage positive for SARS-CoV-2 in Mexico City. COVID-19 Coronavirus disease 2019; $C t$ Cycle threshold; $r R T$-PCR Real-time reverse-transcription polymerase chain reaction
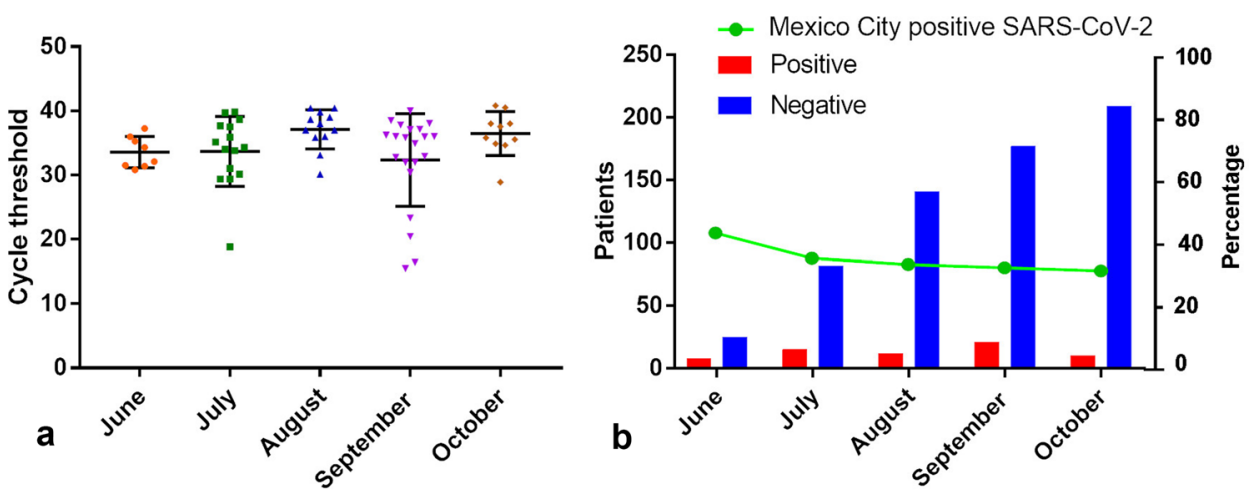
attend follow-up visits and receive scheduled treatments. For example, in Wuhan, the epicenter of the COVID-19 pandemic, the frequency of asymptomatic SARS-CoV-2 infection in a group of children with hemato-oncological diseases was very low (0.33\%) [14]. In New York City, the frequency of infection in 120 asymptomatic cancer patients without known exposure to SARS-CoV-2 was 2.5\% from March 10 to April 12, 2020 [15]. In this crosssectional study, the highest percentage of positivity was observed in children with hemato-oncological diseases (23/177, 12.9\%), followed by children with gastrointestinal diseases $(17 / 174,9.7 \%)$.

This study was conducted at the Hospital Infantil de México Federico Gómez (HIM), which is one of four tertiary pediatric hospitals located in Mexico City. This hospital serves patients who do not have social security, and patients are referred from all over the Mexican Republic. During the pandemic, the Government of Mexico decreed the conversion of this hospital to a designated hospital for the exclusive care of pediatric patients with COVID19 , which necessitated the cessation of clinical care for a significant number of patients who needed medical and surgical management of diseases other than COVID-19. Given the decreasing number of cases of COVID-19 in Mexico City, the hospital restarted care in June 2020 for patients with other diseases, particularly those with hemato-oncological and renal problems. All of the above factors could partly explain why the percentage of positivity for COVID-19 declined from June to October. Fewer children were cared for in the first few months, as the hospital gradually began to admit patients for surgical procedures; 33 children were admitted in June, 97 were admitted in July, and 219 were admitted in October. However, the reduction in the proportion of patients who were positive for SARS-CoV-2 is more likely related to the evolution of the pandemic in Mexico City, as there was a significant decrease in positivity from June (43.92\%) to October (31.09\%), as was also shown in Fig. 1b [4].

Several hospitals have implemented universal screening programs in which all patients, not just patients who will undergo surgery, are screened. Any patient who enters a medical facility could be infected with SARS-CoV-2 and transmit the virus. If aerosol-generating procedures are needed, the risk is increased for all healthcare personnel. According to the results of the study by Poline et al. a systematic screening of children admitted to the hospital should be implemented; in a prospective multicenter study in four tertiary pediatric hospitals in Paris performed between 15 and 30 April 2020, they identified positive SARS-CoV-2 RT-PCR tests in 22 of 438 children (5.0\%) [16]. It is noteworthy that in the present study, $66.6 \%$ of the children with a positive RT-PCR test reported having had contact with an infected household member, while only $3.1 \%$ (22/700) of the children with a positive RT-PCR test reported not having had contact with a household member with COVID-19. Therefore, the epidemiological history of contact could be used to optimize screening programs, and RT-PCR tests could be performed in children at high risk of infection.

It is important to highlight that the frequency of SARS$\mathrm{CoV}-2$ infection in these asymptomatic pediatric patients who visited a pediatric hospital was higher than those reported in other countries with sustained community transmission. However, it needs to be clarified that the group of children who were screened for SARS-CoV-2 in this study do not represent the general pediatric population related to age, health status, immunologic status, or demographic factors. Thus, recognizing the existence of asymptomatic cases reinforces the importance of health care workers using appropriate personal protective equipment, including N95 or equivalent respirators and eye protection when performing aerosol-generating procedures during the pandemic [17-19].

A positive SARS-CoV-2 RT-PCR test in an asymptomatic patient does not imply a high risk of transmission. $\mathrm{Ct}$ values are affected by many variables, such as the number of days from infection to specimen collection, testing method, specimen source, type of transport media, time from collection to analysis, and amount of viral RNA that can be detected by a particular assay, among others. Currently, there is no standardized reference that can be used to validate the $\mathrm{Ct}$ values. While the value could reflect the fact that the patient was in the last days of the infection, it is difficult to arrive at an accurate interpretation based on a single specimen; in the present study, the median Ct value was similar in every month and was always $\leq 36[20,21]$.

As new data emerged every day, medical associations publish the recommendations for the best time of surgery. According to the Association of Anaesthetists, the Center for Peri-operative Care, the Federation of Surgical Specialty Associations, the Royal College of Anaesthetists and the Royal College of Surgeons of England, elective surgery should not be scheduled within $7 \mathrm{wk}$ of a diagnosis of SARS-CoV-2 infection unless the risks of deferring surgery outweigh the risk of postoperative morbidity or mortality associated with COVID-19 [22].

Among the limitations of this study was that the source of infection was difficult to identify; the results cannot be generalized to populations other than children attending this pediatric hospital, both because it was carried out in a tertiary care hospital, and the patient selection process was for convenience. In addition, in some cases, parents were unwilling to disclose all relevant information because they wanted to avoid deferring the procedures. 


\section{Conclusion}

The frequency of asymptomatic infection with SARS-CoV-2 in pediatric patients undergoing invasive medical procedures in a tertiary pediatric hospital was $9.4 \%$. This frequency is higher than those in reports from different countries.

\section{Ethics Approval}

This protocol was evaluated and approved by the National Research and Health Ethics Committee of the Hospital Infantil Federico Gómez (Registry Number HIM-2020-023).

Supplementary Information The online version contains supplementary material available at https://doi.org/10.1007/s12098-021-03959-3.

Authors' Contributions BLM and GBR designed the study and acquired the information. IPO and CERM analyzed and interpreted the data. MAVK and MGMN critically revised the manuscript for important intellectual content, wrote the discussion and approved the final version of the article; MKK, DDLRZ, MVG, JNZ and JGE wrote the discussion and approved the final version of the article; JNZC analyzed and interpreted the data, wrote the article and will act as the guarantor for this paper.

\section{Declarations}

\section{Conflict of Interest None.}

\section{References}

1. WHO Coronavirus (COVID-19) Dashboard. In: World Health Organization (WHO). 2021. Available at: https://covid19.who.int/. Accessed on 24 Dec 2020.

2. Zhu N, Zhang D, Wang W et al. China Novel Coronavirus Investigating and Research Team. A novel coronavirus from patients with pneumonia in China, 2019. N Engl J Med. 2020;382:727-33.

3. Weiss SR, Leibowitz JL. Coronavirus pathogenesis. Adv Virus Res. 2011;81:85-164.

4. COVID-19 Mexico. In: Gobierno de México. 2021. Available at: https://datos.covid-19.conacyt.mx/\#DOView. Accessed on 6 Jan 2021.

5. Cao Q, Chen YC, Chen CL, Chiu CH. SARS-CoV-2 infection in children: transmission dynamics and clinical characteristics. J Formos Med Assoc. 2020;119:670-3.

6. Ludvigsson JF. Systematic review of COVID-19 in children show milder cases and a better prognosis than adults. Acta Paediatr. 2020;109:1088-95.
7. Tang A, Xu W, Shen $\mathrm{M}$ et al. A retrospective study of clinical characteristics of COVID-19 infection in 26 children Med Rxiv. 2020.

8. Han MS, Choi EH, Chang SH, et al. Clinical characteristics and viral RNA detection in children with coronavirus disease 2019 in the Republic of Korea. JAMA Pediatr. 2021;175:73-80.

9. Lauer SA, Grantz KH, Bi Q, et al. The incubation period of coronavirus disease 2019 (COVID-19) from publicly reported confirmed cases: estimation and application. Ann Intern Med. 2020;172:577-82.

10. American College of Surgeons. Joint Statement: Roadmap for Maintaining Essential Surgery during COVID-19 Pandemic. 2020. Available at: https://www.facs.org/covid-19/clinicalguidance/roadmapmaintain-essential-surgery. Accessed on 23 Nov 2020.

11. Ciofi Degli Atti ML, Campana A, Muda AO et al. Facing SARSCoV-2 Pandemic at a COVID-19 Regional Children's Hospital in Italy. Pediatr Infect Dis J. 2020;39:e221-5.

12. Corman VM, Landt O, Kaiser M, et al. Detection of 2019 novel coronavirus (2019-nCoV) by real-time RT-PCR. Euro Surveill. 2020;25:2000045.

13. Okonkwo INC, Howie A, Parry C, et al. The safety of paediatric surgery between COVID-19 surges: an observational study. Anaesthesia. 2020;75:1605-13.

14. Wang SM, Tao F, Hou Y, et al. Screening of SARS-CoV-2 in 299 hospitalized children with hemato-oncological diseases: a multicenter survey in Hubei. China Curr Med Sci. 2020;40:642-5.

15. Boulad F, Kamboj M, Bouvier N, Mauguen A, Kung AL. COVID19 in children with cancer in New York City. JAMA Oncol. 2020;6:1459-60.

16. Poline J, Gaschignard J, Leblanc C, et al. Systematic SARS-CoV-2 screening at hospital admission in children: a French prospective multicenter study. Clin Infect Dis. 2021;72:2215-7.

17. Amicucci M, Mastronuzzi A, Ciaralli I, et al. The management of children with cancer during the COVID-19 pandemic: a rapid review. J Clin Med. 2020;9:3756.

18. Ojetti V, Covino M, Brigida M, et al. Non-COVID diseases during the pandemic: where have all other emergencies gone? Medicina (Kaunas). 2020;56:512.

19. Eke UA, Eke AC. Personal protective equipment in the siege of respiratory viral pandemics: strides made and next steps. Expert Rev Respir Med. 2021;15:441-52.

20. Binnicker MJ. Challenges and controversies to testing for COVID19. J Clin Microbiol. 2020;58:e01695-1720.

21. Rhoads D, Peaper DR, She RC, et al. College of American Pathologists (CAP) microbiology committee perspective: caution must be used in interpreting the cycle threshold $(\mathrm{Ct})$ value. Clin Infect Dis. 2021;72:e685-6.

22. El-Boghdadly K, Cook TM, Goodacre T et al. SARS-CoV-2 infection, COVID-19 and timing of elective surgery: a multidisciplinary consensus statement on behalf of the association of anaesthetists, the centre for peri-operative care, the federation of surgical specialty associations, the royal college of anaesthetists and the royal college of surgeons of England. Anaesthesia. 2021;76:940-6.

Publisher's Note Springer Nature remains neutral with regard to jurisdictional claims in published maps and institutional affiliations. 